\title{
Supervised Machine Learning for Risk Stratification of Influenza-Like Illness: A Model to Prioritize Emerging Infectious Disease Disaster Responses
}

\section{Han-Yi Robert Chiu}

Department of Emergency Medicine, National Taiwan University Hospital and College of Medicine, National Taiwan University, Taipei, Taiwan (100), R.O.C.

\section{Chun-Kai Hwang}

Department of Computer Science and Information Engineering, National Taiwan University, Taipei,

Taiwan (106), R.O.C.

\section{Shey-Ying Chen}

Department of Emergency Medicine, National Taiwan University Hospital and College of Medicine, National Taiwan University, Taipei, Taiwan (100), R.O.C.

\section{Fuh-Yuan Shih}

National Taiwan University Cancer Center, National Taiwan University, Taipei, Taiwan (106), R.O.C.

\section{Hsieh-Cheng Han}

Research Center for Applied Sciences, Academia Sinica, Taipei (115), Taiwan, R.O.C. -Deceased 2020

\section{Chwan-Chuen King}

Institute of Epidemiology and Preventive Medicine, College of Public Health, National Taiwan University, Taipei, Taiwan (100), R.O.C.

\section{John Reuben Gilbert}

Department of Computer Science and Information Engineering, National Taiwan University, Taipei,

Taiwan (106), R.O.C.

\section{Cheng-Chung Fang ( $\square$ conrad@ntu.edu.tw)}

Department of Emergency Medicine, National Taiwan University Hospital and College of Medicine, National Taiwan University, Taipei, Taiwan (100), R.O.C.

\section{Yen-Jen Oyang}

Institute of Biomedical Electronics and Bioinformatics, College of Electrical Engineering and Computer Science, National Taiwan University, Taipei, Taiwan (100), R.O.C.

\section{Research Article}

Keywords: decision trees, deep neural networks, disaster response, emerging infectious disease, influenza-like illness, machine learning, pandemic 
Posted Date: August 13th, 2021

DOl: https://doi.org/10.21203/rs.3.rs-797641/v1

License: (c) (i) This work is licensed under a Creative Commons Attribution 4.0 International License. Read Full License

Version of Record: A version of this preprint was published at Scientific Reports on January 10th, 2022. See the published version at https://doi.org/10.1038/s41598-021-03687-w. 


\section{Front Cover}

2 Supervised Machine Learning for Risk Stratification of Influenza-Like Illness: A

3 Model to Prioritize Emerging Infectious Disease Disaster Responses

4 Han-Yi Robert Chiu ${ }^{1 \#, ~ C h u n-K a i ~ H w a n g " \#, ~ S h e y-Y i n g ~ C h e n ~}{ }^{1}$, Fuh-Yuan Shih",3, Hsieh-Cheng

5 Han $^{4 \dagger}$, Chwan-Chuen King ${ }^{5}$, John Reuben Gilbert ${ }^{2}$, Cheng-Chung Fang ${ }^{*}$, and Yen-Jen Oyang,6*

$6{ }^{1}$ Department of Emergency Medicine, National Taiwan University Hospital and College of Medicine,

$7 \quad$ National Taiwan University, Taipei, Taiwan (100), R.O.C.

$8{ }^{2}$ Department of Computer Science and Information Engineering, National Taiwan University, Taipei,

9 Taiwan (106), R.O.C.

$10{ }^{3}$ National Taiwan University Cancer Center, National Taiwan University, Taipei, Taiwan (106), R.O.C.

$11{ }^{4}$ Research Center for Applied Sciences, Academia Sinica, Taipei (115), Taiwan, R.O.C.

$12{ }^{5}$ Institute of Epidemiology and Preventive Medicine, College of Public Health, National Taiwan

13 University, Taipei, Taiwan (100), R.O.C.

$14{ }^{6}$ Institute of Biomedical Electronics and Bioinformatics, College of Electrical Engineering and Computer

15 Science, National Taiwan University, Taipei, Taiwan (100), R.O.C.

16 \# These authors contributed equally to this work * Corresponding Authors $\quad$ Deceased 


\section{Contact information of Corresponding Authors}

2

3 Yen-Jen Oyang: Ph. D.

4 Inst. of Biomedical Electronics and Bioinformatics,

5 College of Electrical Engineering and Computer Science,

6 National Taiwan University

7 No. 1, Sec. 4, Roosevelt Road

8 Taipei, Taiwan (106), Republic of China (R.O.C.)

9 Tel: +886-2-33664888 ext. 431; Fax: +886-2-23688675;

10 E-mail: yjoyang@csie.ntu.edu.tw

11

12 Cheng-Chung Fang, M. D.

13 Department of Emergency Medicine,

14 National Taiwan University Hospital,

15 No. 7 Chung Shan S. Road

16 Taipei, Taiwan (100), Republic of China (R.O.C.)

17 Tel: +886-2-23123456 ext. 65629; Fax: +886-2-23223150;

18 E-mail: conrad@ntu.edu.tw

19

20 Running Title: Machine Learning Model for EID Responses

21

22 Key words: decision trees, deep neural networks, disaster response, emerging infectious disease,

23 influenza-like illness, machine learning, pandemic 


\section{Cover Letter}

\section{Richard White}

3 scientificreports@nature.com

5 Dear Editors-in-Chief (Professors Richard White):

August 09, 2021

6 My colleagues and I would like to submit an article entitled "Supervised Machine Learning for Risk

7 Stratification of Influenza-Like Illness: A Model to Prioritize Emerging Infectious Disease Disaster

8 Response" for your consideration as an original research article in Scientific Reports.

9 Our study was motivated by the observation that emerging infectious diseases (EIDs), including the latest COVID-19, have led to several global medical and public health disasters. An EID may spread rapidly and collapse medical facilities due to the medical demand far exceeding their capacity. Consequently, it is vital to allocate patients with different disease severity risks to optimize the utilization of medical resources. In this study, we aimed to combat this challenge with machine learning. This inter-disciplinary study was based on a national health insurance database collected in Taiwan. We analyzed 83,227 subjects admitted with influenza-like illness for risk of fatality or requiring critical cares. The risk factors considered included age, gender, and 19 comorbidities. We investigated the performance delivered by three different types of prediction models, namely the decision tree (DT) models, the deep neural networks (DNN) models, and the logistic regression models. Our results showed that the DT prediction models built with only 6 features delivered performance comparable to the state-of-the-art DNN prediction models. As the structures of the DT models explicitly demonstrated the prediction logic, we have concluded that DT models can be easily adapted by clinicians to provide high-risk patients with the best medical treatments. Furthermore, the DT models with alternative sensitivity levels can be applied in different stages of an EID disaster to effectively optimize medical resource allocation.

There is no conflict of interest in the submission of this manuscript, and the manuscript has been approved by all authors for publication. On behalf of all co-authors, we declare that the work described is original 27 in whole or in part.

28 We deeply appreciate your consideration of our manuscript for publication, and we look forward to 29 receiving comments from the reviewers. If you have any questions, please do not hesitate to contact us 30 at the following addresses.

31 Sincerely, 


\section{Yen-Jen Oyang, Ph. D.}

2 Inst. of Biomedical Electronics and Bioinformatics,

3 College of Electrical Engineering and Computer Science,

4 National Taiwan University, Room \#431, Der-Tian Hall,

5 No. 1, Sec. 4, Roosevelt Road

6 Taipei, Taiwan (106), Republic of China (R.O.C.)

7 Tel: +886-2-33664888 ext. 431; Fax: +886-2-23628167;

8 E-mail: yjoyang@csie.ntu.edu.tw

9

\section{Cheng-Chung Fang, M. D.}

11 Department of Emergency Medicine,

12 National Taiwan University Hospital,

137 Chung Shan S. Road

14 Taipei, Taiwan (100), Republic of China (R.O.C.)

15 Tel: +886-2-23123456 ext. 65629; Fax: +886-2-23223150;

16 E-mail: conrad@ntu.edu.tw

17

18

19

20 


\section{Abstract}

2 Emerging infectious diseases (EIDs), including the latest COVID-19 pandemic, have emerged and raised

3 global public health crises in recent decades. Without existing protective immunity, an EID may spread

4 rapidly and cause mass casualties in a very short time. Therefore, it is imperative to identify cases with

5 risk of disease progression for the best allocation of medical resources in case medical facilities are

6 overwhelmed with a flood of patients. This study aimed to exploit machine learning technologies to cope

7 with this challenge. The study was based on 83,227 hospital admissions with influenza-like illness and

8 we analysed the risk effects of 19 comorbidities along with age and gender for severe illness or mortality

9 risk. The experimental results revealed that the conventional decision tree (DT) models built with only 6

10 features, including age, gender, and four comorbidities, delivered the same level of prediction accuracy

11 as the state-of-the-art deep neural network models built with 18 features. Accordingly, we further studied

12 how to exploit the DT models with different sensitivity levels to determine patient triage and optimize

13 medical resource allocation in different stages of an EID disaster to aid the frontline clinicians and policy-

14 makers. In conclusion, our study demonstrated an approach to exploit machine learning technologies to

15 cope with the challenges during the outbreak of an EID. 


\section{Introduction}

2 Emerging infectious diseases (EIDs), including the severe acute respiratory syndrome (SARS) (2003) ${ }^{1}$,

3 H1N1 influenza virus (2009) $)^{2}$, Middle East respiratory syndrome coronavirus (MERS-CoV) $(2013)^{3}$, and

4 coronavirus disease 2019 (COVID-19) pandemic ${ }^{4}$, emerged and raised global public health crises in

5 recent decades. Without existing protective immunity at both individual and population levels, an

6 emerging infectious disease may spread efficiently and lead to massive severe cases and mortality in the

7 community $^{5}$. In particular, with a highly contagious novel respiratory infectious disease ${ }^{6}$, medical

8 resources, including medications, personal protective and life-supporting equipment, may be quickly

9 exhausted once hospitals are overwhelmed with infected patients ${ }^{7,8}$. It may inevitably cause excessive

10 mortality as demonstrated in many countries during the 2020-2021 COVID-19 pandemic ${ }^{8,9}$. As the

11 clinical spectrum of emerging respiratory infections may range from asymptomatic or mild respiratory

12 symptom to severe pneumonia or acute respiratory distress syndrome ${ }^{10,11}$, it is therefore imperative for

13 first-line physicians to prioritize scarce medical resources for critically ill patients and early symptomatic

14 patients with high risk of rapid progression and death ${ }^{9,12}$. However, in the early stage of the outbreak of

15 a novel respiratory infectious disease, there is usually no prior knowledge and available guidelines for

16 the physicians to optimize medical decisions. Accordingly, it is of interest to investigate how to exploit

17 machine learning (ML) technologies to cope with this challenge.

18 In recent years, ML technologies have been widely exploited in medical and public health research ${ }^{12-14}$.

19 ML algorithms are highly effective in analyzing interactions among multiple, complex variables in

20 clinical databases and making accurate predictions, which may take a medical practitioner months or

21 even years to accumulate sufficient experience to develop a decision making process. However, there are

22 a wide range of ML algorithms with very different characteristics and design goals. At one end of the

23 spectrum, advanced ML algorithms such as the deep neural network (DNN) ${ }^{14,15}$ and the support vector

24 machine $(\mathrm{SVM})^{16}$ employ complicated non-linear transformations to achieve superior prediction

25 accuracy. However, due to the complicated non-linear transformations involved, it is essentially

26 impossible to figure out how these kinds of ML algorithms make predictions. At the other end of the

27 spectrum, ML algorithms such as decision trees (DT) ${ }^{17-19}$ and the naïve Bayesian classifier follow highly

28 interpretable decision processes to make predictions ${ }^{20}$ but may suffer inferior prediction accuracy due to

29 lack of non-linear transformations involved in the prediction process. The trade-off between prediction

30 accuracy and interpretability with alternative ML algorithms may be an everlasting dilemma depending

31 on different clinical applications. As pointed out by Flaxman and Vos, for some applications, using an

32 explainable approach is more understandable and favourable for physicians even when it results in a 
1 slight reduction in accuracy ${ }^{20}$.

2 In this study, we conducted a comprehensive investigation on how ML algorithms can be exploited to

3 cope with the challenges addressed above. We analyzed how different types of prediction models, namely 4 the conventional decision tree (DT) ${ }^{21,22}$ prediction models, the state-of-the-art Deep Neural Network 5 (DNN) prediction models ${ }^{15}$, as well as the logistic regression (LR) based prediction models, performed 6 in identifying the patients with high risk of rapid progression and death. With the experimental results, 7 our discussions have focused on how DT prediction models can be incorporated to facilitate physicians' 8 decisions on triaging patients based on risk of clinical deterioration and prioritizing scarce medical 9 resources for best population outcomes during a pandemic of EID. 


\section{Methods}

\section{Data Collection and Outcome Measurement}

3 We conducted this study based on the reimbursement data of one million randomly sampled subjects

4 extracted from the de-identified National Health Insurance Research Database (NHIRD) in Taiwan.

5 Figure 1 shows the process to generate the cohort. We began with 92,376 hospitalized ILI cases during

6 January 2005 to December 2010. Supplementary Table S1 lists the ICD-9-CM (International

7 Classification of Diseases, $9^{\text {th }}$ Revision, Clinical Modification) codes employed to define an ILI case,

8 which were identified through syndromic surveillance ${ }^{23}$ and intensive discussions among Taiwanese

9 physicians ${ }^{24,25}$. The information retrieved from ILI patients' records included age, gender, and 19

10 comorbidities/conditions [heart disease, peripheral vascular disease, hypertension, cerebrovascular

11 accident (CVA), neurological disease, pulmonary disease, allergic rhinitis, autoimmune disease, liver

12 disease, diabetes, hyperthyroidism, hypothyroidism, renal disease, metastatic cancer, cancer without

13 metastasis, leukaemia/lymphoma, acquired immunodeficiency disease, tuberculosis, mental illness, and

14 pregnancy/postpartum women]. These comorbidities were identified based on a literature review and

15 thorough consensus reached by physicians of infection, emergency medicine, occupational health and

16 infectious disease epidemiologists ${ }^{26}$. The corresponding ICD-9-CM codes employed to identify the 19

17 comorbidities are shown in Supplementary Table S2, which were defined based on the Charlson ${ }^{27,28}$,

18 Deyo $^{29}$ and Elixhauser ${ }^{30}$ measurements plus information from the Taiwanese Catastrophic Illness Card.

19 Presence of a comorbidity was defined based on whether the patient was coded with the corresponding

20 ICD-9-CM codes within 12 months prior to the index date of the ILI-related hospitalisation.

21 With the initial 92,376 hospitalized ILI cases, we excluded 250 cases with unrecognized identity, that

22 absconded from hospitals, or committed suicide and additional 2,687 cases with incomplete records.

23 Then, we merged two consecutive records of the same patient if these two consecutive records were

24 within 14 days. In the end, a cohort containing 83,227 cases was created (Figure 1).

25 The outcome of concern was severe ILI, which was defined as the occurrence of fatality or requiring

26 critical cares such as intubation, ventilator support, extracorporeal membrane oxygenation treatment,

27 admission to an intensive care unit during the hospitalization period. This study was approved by the

28 Research Ethics Committee of National Taiwan University Hospital (\#201603086RINB). 


\section{Experimental Procedures}

2 Figure 2 shows the experimental procedure employed in this study to analyze the performance delivered

3 by different types of prediction models. The analysis began with a 2-stage feature selection process. In

4 the first stage, we employed the conventional logistic regression (LR) analysis to eliminate those features

5 that were uncorrelated to the outcome variable. Then, in the second stage, two advanced multivariate

6 analysis methods, namely being the least absolute shrinkage and selection operator (LASSO) method ${ }^{31}$

7 and the ensemble variant of minimum redundancy maximum relevance (mRMRe) method ${ }^{32,33}$, were

8 employed along with the proposed DT-based method to determine the minimal subsets of the features

9 without compromising prediction performance. With the three feature sets output by the LASSO, the 10 mRMRe, and the proposed DT-based method, we proceeded to build the $\mathrm{DT}^{17-19}$, the $\mathrm{LR}^{34}$, and the deep

11 neural network (DNN) ${ }^{15}$ prediction models. Finally, the performance of these different prediction models 12 were evaluated using the 10 -fold cross validation ${ }^{35}$.

\section{Feature Selection}

14 The feature selection process began with the 21 -variable feature set shown in Table 1 . Table 1 also shows

15 the results of the first-stage LR based analysis. Since the p-values with mental illness, hypothyroidism,

16 and hyperthyroidism were higher than 0.05 , these three comorbidities were excluded. With the remaining

1718 variables, we proceeded to carry out the DT-based multivariate analysis proposed in this study. In this

18 procedure, the DT package shown in Supplementary Table S3 was employed and the prior and cp

19 parameters ${ }^{18}$ were set to different values in order to generate models with various sensitivity levels.

20 Supplementary Table S4 shows the DT models that delivered sensitivity at the 85\%, 90\%, and 95\% levels,

21 respectively. Then, we selected the 6 variables that were consistently present in all of these DT models.

22 To evaluate the effectiveness of the proposed DT-based multivariate analysis, we further incorporated

23 the $\mathrm{LASSO}^{31}$ and the mRMRe ${ }^{32,33}$ methods to extract another two 6-variable feature sets from the 18-

24 variable feature set output by the first-stage feature selection process. Then, we proceeded to build the 
1 DT models, the LR models, and the DNN models based on these three 6-variable feature sets for

2 performance evaluation.

\section{The Development of Prediction Models}

4 In this study, we developed two types of machine learning-based prediction models, namely being $\mathrm{DT}^{17-}$

$5{ }^{19}$ and DNN models ${ }^{15}$. The performance of the DT models is of interest due to the explicit decision rules

6 produced by the DT algorithm, which is a unique feature favored by clinicians. However, the algorithm

7 for building a DT model is based on univariate analysis and does not incorporate any linear or non-linear

8 transformation. As a result, the prediction performance of the DT models may not match the advanced

9 prediction models when applied to those datasets in which different classes of samples are separated by

10 non-linear boundaries. In this respect, with the advantage of non-linear transformations, the state-of-the-

11 art DNN models generally can deliver superior prediction performance in comparison with other types

12 of prediction models ${ }^{36}$. However, a DNN based model typically contains a large quantity of coefficients

13 and therefore it is almost impossible for clinicians to figure out the logic embedded in the prediction

14 process. In this study, we further investigated how the conventional LR models ${ }^{34,37}$ performed because

15 logistic regression is widely used in medical and epidemiological research. Supplementary Table S3

16 summarizes the software packages and parameter settings employed to build the DT models and the main

17 characteristics of the DNN models. With respect to the structure of the DNN models, we actually

18 investigated the performance of more complicated networks and observed that the simple network

19 structure shown in Supplementary Table S3 delivered the same level of performance in comparison with

20 more complicated network structures. In this respect, we experimented with network dimensions of 8 ,

2116,24 and 32 and set the number of layers to 3 and 4.

\section{Model Performance Evaluation}

23 To evaluate model performance, we employed 10-fold cross validation to evaluate the performance of 24 our prediction models. ${ }^{35}$ As shown in Supplementary Table S3, in order to generate the DT models with 
1 alternative performance characteristics, e.g. different levels of sensitivity, we set the prior and cp

2 parameters ${ }^{18}$ to various values. For generating the LR models and the DNN models with alternative

3 performance characteristics, we varied the cutoff values at the outputs in order to discretize the numerical

4 outputs into binary states. Model performance was evaluated based on several metrics, including

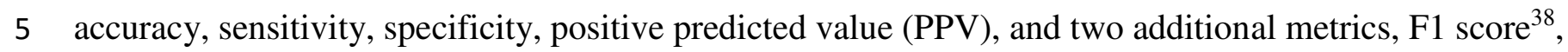

6 and Matthews correlation coefficient $(\mathrm{MCC})^{38}$ designed to report the overall performance of the

7 prediction models (Supplementary Table S5). The F1 score is defined to be the the harmonic mean of the

8 PPV and the sensitivity delivered by a prediction model and is a widely used performance metric in the

9 machine learning research community. In recent years, the scientists in the biomedical research

10 communities have also started to incorporate the F1 score to report their performance data ${ }^{39}$. 


\section{Results}

2 To conduct a comprehensive performance analysis, we built different types of prediction models with

3 alternative feature sets, Table 2 summaries the F1 scores delivered by these prediction models and the

4 comprehensive performance data is shown in Supplementary Tables S6 (a)-(c). The alternative feature

5 sets incorporated to build the prediction models included the three 6-variable feature sets identified by

6 the proposed DT-based analysis, the mRMRe, and the LASSO, along with the 18-variable feature set

7 identified by the logistic regression based analysis in the first stage of the feature selection process.

8 With respect to performance data shown in Table 2 and Supplementary Tables S6 (a)-(c), the first

9 observation was that the DNN model built with 18 variables performed marginally superior to the other

10 prediction models shown in Table 2. For example, under the column of $85 \%$ sensitivity, the F1 score of

110.452 delivered by the DNN model built with 18 variables was marginally higher than the other F1 scores

12 delivered by the three DNN models built with the three different 6-variable feature sets, which were

$130.447,0.438$, and 0.437 , respectively. This observation implied that no significant information was lost

14 when we employed only 6 variables. The second observation was that all these different types of

15 prediction models built with alternative 6-variable feature sets basically delivered the same level of

16 performance. For example, under the column with $85 \%$ sensitivity, the F1 scores delivered by different

17 prediction models built with different 6-variable feature sets were all within the range from 0.433 to

18 0.447. Accordingly, in the following discussion, we focused on the DT models built with 6 variables

19 because the explicit prediction logic output by the DT algorithm was highly valuable with respect to

20 clinical applications. The third observation was that the DT models built with the 6-variable feature set

21 identified by the proposed DT-based method performed marginally superior to the DT models built with

22 the 6-variables features sets identified by the mRMRe and the LASSO. For example, under the column

23 with $85 \%$ sensitivity, the F1 scores delivered by the DT models built with the 6-variables features sets 
1 identified by the proposed DT-based method, the mRMRe, and the LASSO were 0.446, 0.438, and 0.437,

2 respectively.

3 As decision-makers like to know how to allocate resources most appropriately under different scenarios,

4 Figure 3 (a)-(c) shows the DT models that delivered 95\%, 90\%, and 85\% sensitivities, respectively. Since

5 age was placed at the top level of the tree structures in all these three models, it implied that age was the

6 most crucial factor. The DT model with $95 \%$ sensitivity revealed that patients aged over 37.79 or under

70.54 years suffered high risk for severe ILI. Furthermore, the following two groups of patients also

8 suffered high risk for severe ILI: (1) patients aged between 14.21 and 37.79 with heart disease, CVA,

9 diabetes, metastatic cancer; and (2) male gender aged between 34.46 and 37.79 (Figure 3 (a)). The DT

10 model with $90 \%$ sensitivity revealed that those patients older than 66.04 years-old suffered the highest

11 risk of progression to severe illness. Furthermore, those female patients aged between 41.46 and 66.04

12 and with CVA, diabetes, heart disease, and metastatic cancer also suffered high risk for severe ILI (Figure

133 (b)). The DT model with $85 \%$ sensitivity identified the following three groups of patients that suffered

14 high risk of severe ILI: (1) patients older than 66.04; (2) male patients aged between 41.46 and 66.04

15 with heart disease, metastatic cancer, CVA, and diabetes; and (3) female patients aged between 41.46

16 and 66.04 and with CVA (Figure 3 (c)). Overall, 31.0\% (25780/83227), 41.7\% (34681/83227) and 48.3\%

17 (40187/83227) of those hospitalized ILI patients were predicted to have low risk of progression to severe

18 ILI by the three DT models with $95 \%, 90 \%$ and $85 \%$ sensitivity, respectively (Figure 3). 


\section{Discussion}

2 We have conducted a comprehensive analysis on how to exploit machine learning algorithms to stratify

3 the risk of severe illness or death among hospitalized ILI patients. There were three major findings in

4 this study. Firstly, the three different types of prediction models investigated in this study, namely the

5 DNN models, the LR models, and the proposed DT based models, delivered comparable performance in

6 predicting severe ILI after hospitalization. Secondly, the tree structures of the DT models explicitly

7 illustrated how predictions were made and provide valuable guidelines for clinicians to develop effective

8 strategies for risk stratification of ILI patients. Thirdly, the clinicians can employ the DT models with an

9 appropriate sensitivity levels to cope with the availability of medical resources and public health needs

10 in different epidemic stages of an EID disaster.

11 With respect to the performance of the different types of prediction models, namely the DT models, the

12 LR models, and the DNN models, our results may be confusing for some machine learning experts who

13 strongly believe that the DNN models should prevail in most cases ${ }^{17,36,40}$. However, how the DNN model

14 performs in comparison with different types of prediction models really depends on how different classes

15 of subjects, e.g. positives and negatives, distribute in the dataset. If different classes of subjects can be

16 partitioned by linear geometric objects defined by a very limited number of features, then different types

17 of prediction models may deliver comparable performance. In other words, the DNN models may not

18 prevail in this case, which was exactly what we observed in this study. In fact, we also observed a similar

19 result from one of our recent study on dengue ${ }^{13}$.

20 With the DT models being able to deliver performance comparable to the state-of-the-art DNN models,

21 the explicit prediction rules presented in the DT structures provide valuable references for developing

22 effective clinical strategies. All the studied DT models with different sensitivities identified age seniority

23 as the most critical risk factor for severe ILI. This result is in conformity with clinical experiences as age 
1 seniority, along with comorbid medical conditions such as diabetes ${ }^{2,26}$, $\operatorname{cirrhosis}^{41}$, malignant diseases ${ }^{26,42}$,

2 etc., has been recognized as one of the crucial risk factors for severe ILI. Furthermore, the cutoffs

3 employed by the DT models to partition age groups are in conformity with clinical insights. Nevertheless,

4 these cutoffs along with the comorbidities identified in the DT structures provide clinicians with

5 systematic clues regarding how to treat the patients most effectively when facing an EID.

6 The DT models with different levels of sensitivity can be employed by the first-line clinicians and policy-

7 makers for modifying patient's triage and resource allocation in different stages of an EID disaster

8 (Figure 4). In the early stage of an EID disaster, when the healthcare capacity is adequate, the DT models

9 with high sensitivity levels should be employed to identify patients with risk of disease progression so

10 that they may be hospitalized and receive early and the best treatment ${ }^{9,43}$ to minimize deaths. As the

11 development of the EID disaster progresses, the tremendous increase of patient number and the demand

12 of medical resources may rapidly exceed the surging capacity of medical facilities. In this scenario,

13 clinicians may be forced to discharge patients with mild symptoms at the early stage of infection

14 irrespective of their potential risk for subsequent deterioration ${ }^{44}$. Accordingly, the DT models with a

15 lower sensitivity should be employed to identify only those patients with high risk to progress to severe

16 infection or even death for hospitalization and therefore avoid collapse of medical facilities. For example,

17 with the DT model that delivered $85 \%$ sensitivity in our study, $48.3 \%$ of the admitted ILI patients might

18 be released from medical facilities. This strategy is of particular value in EID pandemic such as the

19 COVID-19 outbreaks since nearly all countries with community outbreaks experienced unprecedented

20 mortality due to collapse of the healthcare system.

21 There are several limitations in the current study. Firstly, the diagnosis of ILI was based on ICD-9-CM

22 codes without laboratory confirmation of influenza. Nevertheless, ILI-related clinical syndromes may be

23 the best surrogate diagnostic category representative of patients with community-onset respiratory

24 infections that may progress towards severe illness and death ${ }^{24,26}$. Secondly, other potential confounding 
1 factors that may influence the prognosis of respiratory infections, including obesity ${ }^{45,46}$, smoking ${ }^{47}$,

2 geographic distributions ${ }^{48}$, social economic conditions ${ }^{49}$, and the use of antiviral treatments ${ }^{50,51}$ were not

3 available in the NHIRD database. Thirdly, we did not investigate the performance of other advanced

4 machine learning algorithms such as the support vector machine, random forests, Bayesian networks, etc.

5 Nevertheless, it is generally observed that the DNN based prediction models can deliver comparable or

6 ever superior performance when compared with other advanced machine learning algorithms. Fourthly,

7 as our experimental data was extracted from a single national insurance reimbursement database, readers

8 should be cautious to generalize our findings before further validation studies are conducted.

9 In conclusion, our results showed that the DT-based prediction models delivered performance 10 comparable to the DNN models in predicting ILI severity. The explicit prediction logic shown in the DT

11 structures may be exploited to facilitate the decision-making process executed by clinicians. Furthermore,

12 the DT models with alternative sensitivity levels can be exploited in different stages of an EID disaster

13 to optimize medical resource allocation, which is crucial in the response to a large-scale epidemic of 14 emerging infectious disease. 


\section{Figure legends}

2 Figure 1. Flow diagram of the selection process of study subjects.

3 Abbreviations: ICD-9-CM (International Classification of Diseases, $9^{\text {th }}$ Revision, Clinical Modification); ILI

4 (influenza-like illness); NHIRD (National Health Insurance Research Database).

5 Figure 2. The experimental procedures.

6 The analysis began with a 2-stage feature selection process. In the first stage, the conventional logistic

7 regression analysis was employed to eliminate those features that were uncorrelated to the outcome

8 variable. In the second stage, the proposed DT-based method along with two advanced multivariate 9 analysis methods, namely being the least absolute shrinkage and selection operator (LASSO) method $^{31}$ and the ensemble variant of minimum redundancy maximum relevance (mRMRe) $\operatorname{method}^{32,33}$, were employed to generate three 6-variable feature sets. Then, these three 6-variable feature sets were employed to build the DT, LR, and DNN prediction models. Finally, the performance of the alternative prediction models was evaluated based on the 10 -fold cross validation process.

*Abbreviations: CVA (cerebrovascular accident).

Figure 3. Decision trees generated with 6 features at three sensitivities $(95 \%, 90 \%, 85 \%)$.

Figure 3 (a). The decision tree built with the 6 features identified by the proposed DT-based method and delivering $95 \%$ sensitivity.

Abbreviations: TP (true positive); TN (true negative); PPV (positive predictive value) $=$

$\mathrm{TP} /(\mathrm{TP}+\mathrm{FP})$; NPV (negative predictive value) $=\mathrm{TN} /(\mathrm{TN}+\mathrm{FN})$.

Figure 3 (b). The decision tree built with the 6 features identified by the proposed DT-based method and delivering $90 \%$ sensitivity.

24 Note: positive predictive value $(\mathrm{PPV})$, precision $=\mathrm{TP} /(\mathrm{TP}+\mathrm{FP})$; negative predictive value $(\mathrm{NPV})=$ $25 \mathrm{TN} /(\mathrm{TN}+\mathrm{FN}) ; \mathrm{TP}$ : true positive; TN: true negative.

Figure 3 (c). The decision tree built with the 6 features identified by the proposed DT-based method and delivering $85 \%$ sensitivity. 
1 Figure 4. The curve that shows how the number of cases increases as the EID disaster

2 progresses. ${ }^{52}$ Beyond time point of a crisis (Tc), the medical facilities start to operate under a

3 crisis mode.

4 In the early stage of an EID disaster, when the healthcare capacity is adequate, the DT models with

5 high sensitivity levels should be employed to identify patients with risk of infection progression for

6 them to be hospitalized and receive the best treatment ${ }^{9,43}$. In the later stages of an EID disaster, the

7 available medical resources may be exhausted due to a tremendous increase of patients. In this

8 scenario, the DT models with a lower sensitivity should be employed to recommend only those

9 patients with a high risk of progressing to severe infection or death for hospitalization and thereby 10 avoid collapse of medical facilities.

11 
Hospitalized cases with one or more ILI related ICD-9-CM codes

during Jan. 2005 to Dec. 2010 in the NHIRD $(\mathrm{N}=92,376)$

\section{Exclusion Criteria:}

Unrecognized identity, absconding from hospitals, suicide

$(\mathrm{N}=250)$.

Incomplete recordings (e.g. Hospitalization without

discharge date, $\mathrm{N}=2,687$ )

Cases with complete records $(\mathrm{N}=89,439)$

The cohort used in this study $(\mathrm{N}=83,227)$

Two consecutive ILI records of the same patient within14

days were merged $(\mathrm{N}=6,212)$.

ᄂ

2 Figure 1. Flow diagram of the selection process of study subjects.

3 Abbreviations: ICD-9-CM (International Classification of Diseases, $9^{\text {th }}$ Revision, Clinical

4 Modification); ILI (influenza-like illness); NHIRD (National Health Insurance Research Database). 


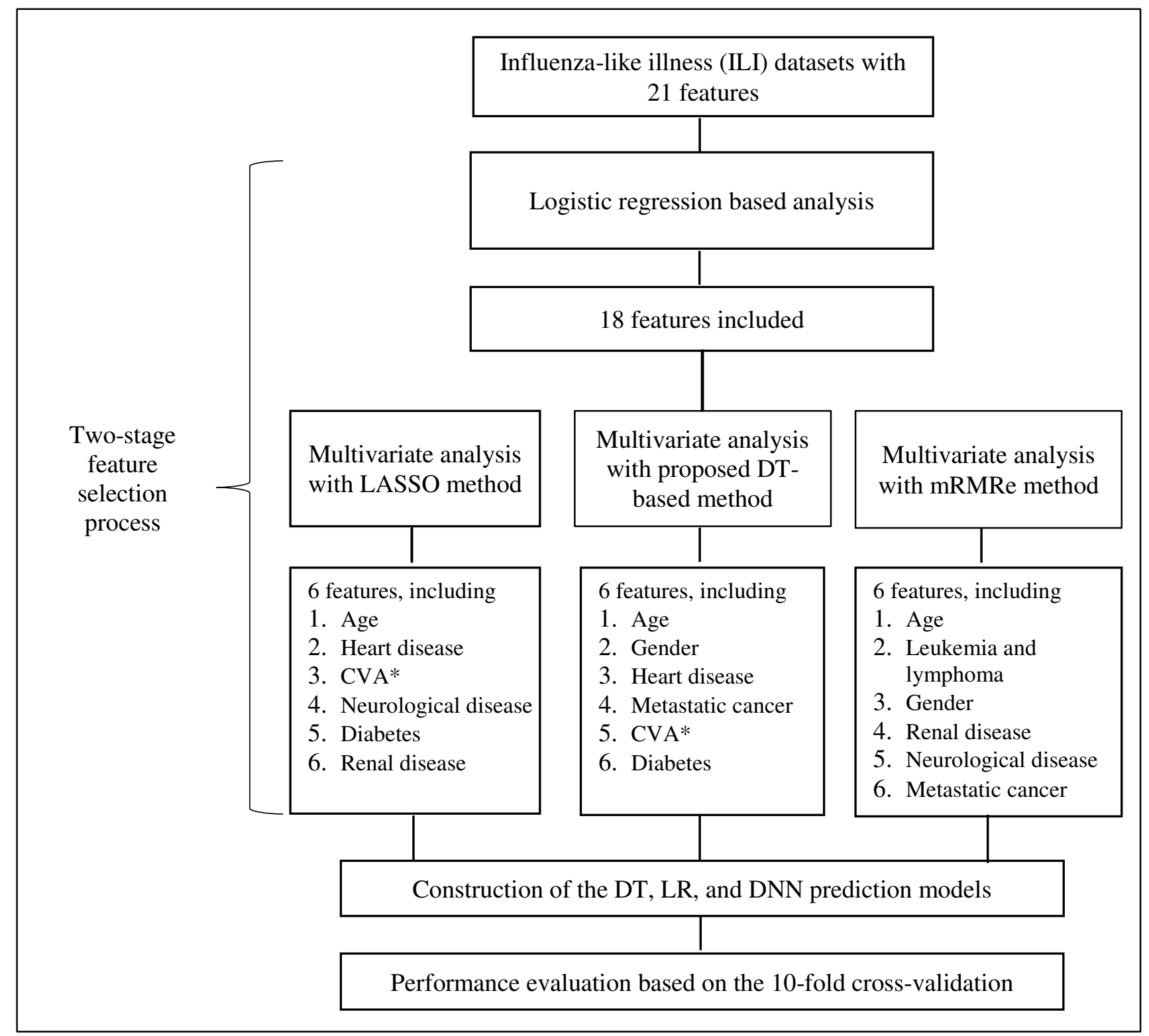

2 Figure 2. The experimental procedures.

3 The analysis began with a 2-stage feature selection process. In the first stage, the conventional logistic 4 regression analysis was employed to eliminate those features that were uncorrelated to the outcome 5 variable. In the second stage, the proposed DT-based method along with two advanced multivariate 6 analysis methods, namely being the least absolute shrinkage and selection operator (LASSO) method ${ }^{31}$ 7 and the ensemble variant of minimum redundancy maximum relevance (mRMRe) method ${ }^{32,33}$, were 8 employed to generate three 6-variable feature sets. Then, these three 6-variable feature sets were 9 employed to build the DT, LR, and DNN prediction models. Finally, the performance of the alternative 10 prediction models was evaluated based on the 10-fold cross validation process.

11 *Abbreviations: CVA (cerebrovascular accident). 


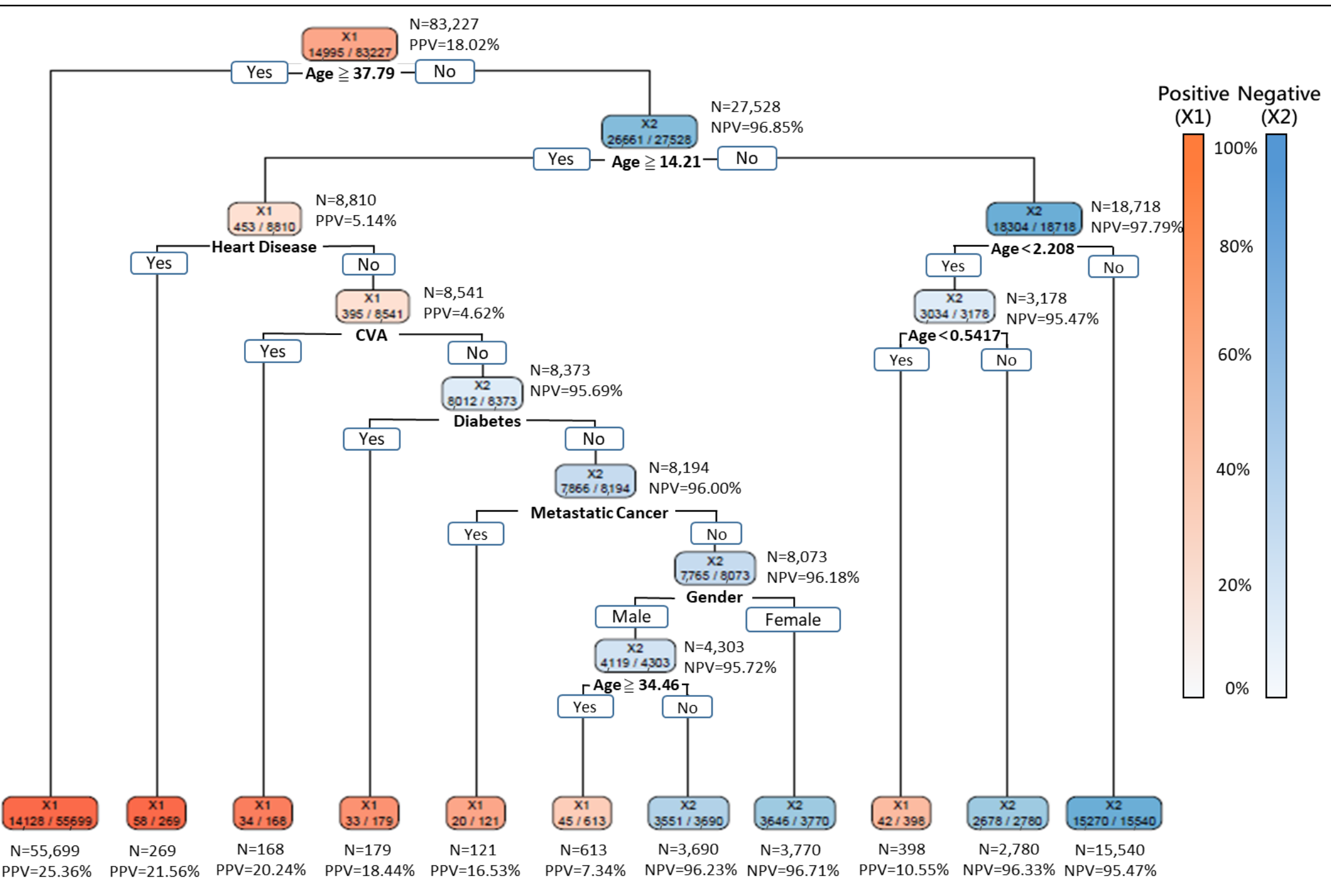

$2 \quad$ Figure 3 (a). The decision tree built with the 6 features identified by the proposed DT-based method and delivering $95 \%$ sensitivity.

3 Abbreviations: $\mathrm{TP}($ true positive); $\mathrm{TN}$ (true negative); $\mathrm{PPV}$ (positive predictive value $)=\mathrm{TP} /(\mathrm{TP}+\mathrm{FP}) ;(\mathrm{NPV}$ (negative predictive value $)=\mathrm{TN} /(\mathrm{TN}+\mathrm{FN})$. 


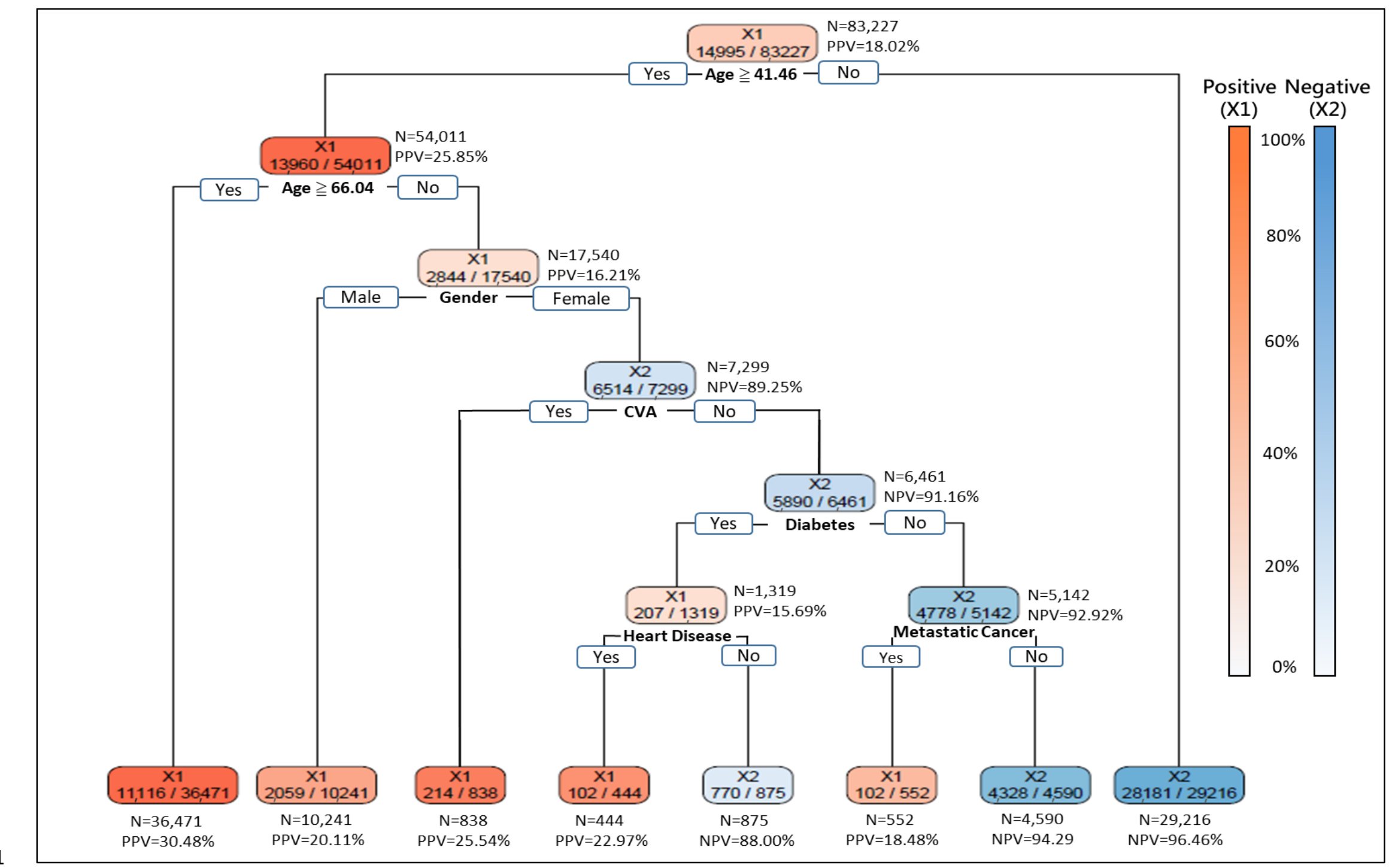

$2 \quad$ Figure 3 (b). The decision tree built with the 6 features identified by the proposed DT-based method and delivering $90 \%$ sensitivity.

3 Abbreviations: $\mathrm{TP}($ true positive); $\mathrm{TN}($ true negative); $\mathrm{PPV}$ (positive predictive value $)=\mathrm{TP} /(\mathrm{TP}+\mathrm{FP}) ; \mathrm{NPV}$ (negative predictive value $)=\mathrm{TN} /(\mathrm{TN}+\mathrm{FN})$. 


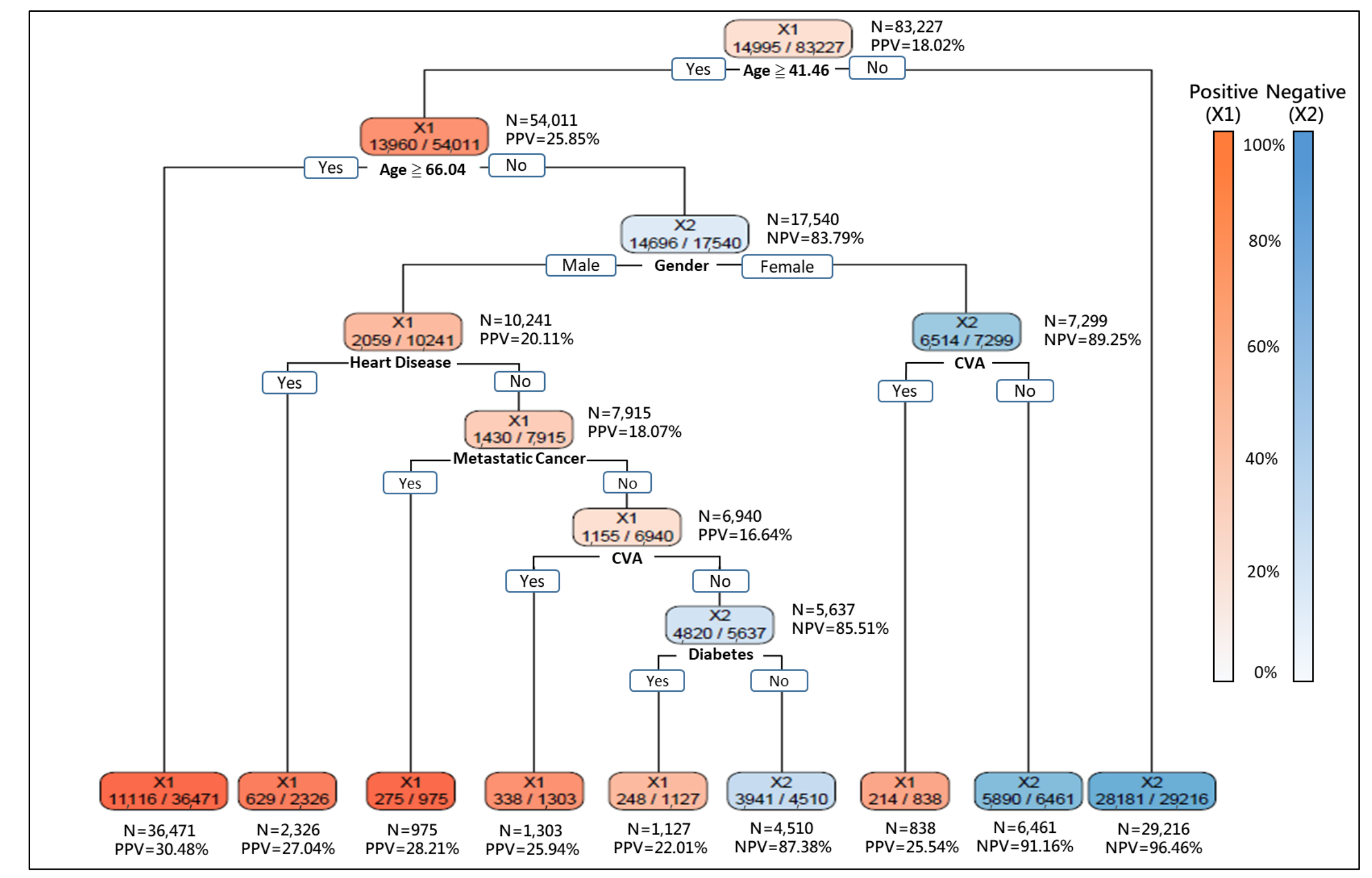

2 Figure 3 (c). The decision tree built with the 6 features identified by the proposed DT-based method and delivering $85 \%$ sensitivity.

3 Abbreviations: $\mathrm{TP}($ true positive); $\mathrm{TN}$ (true negative); $\mathrm{PPV}$ (positive predictive value $)=\mathrm{TP} /(\mathrm{TP}+\mathrm{FP}) ; \mathrm{NPV}($ negative predictive value $)=\mathrm{TN} /(\mathrm{TN}+\mathrm{FN})$. 


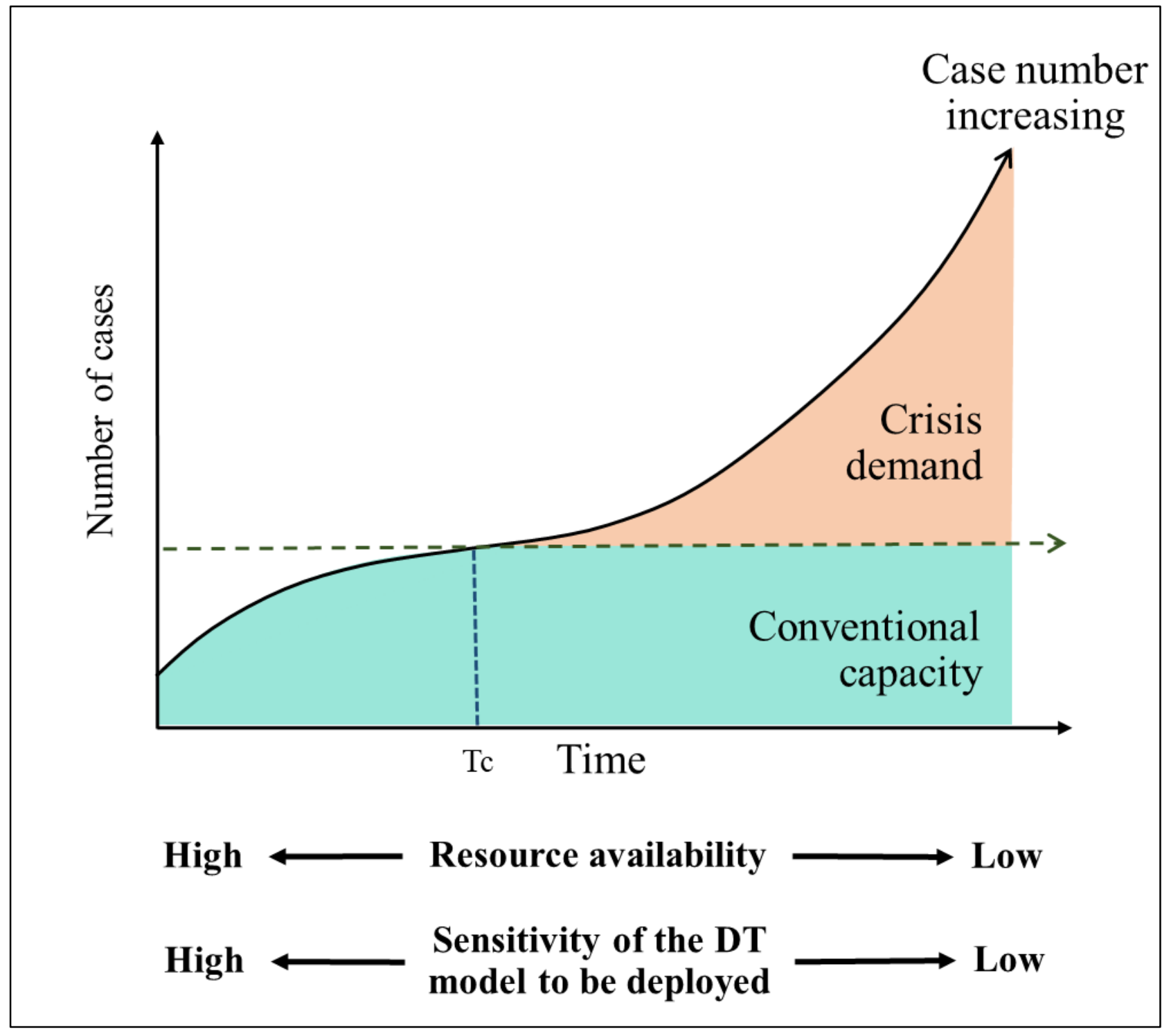

2 Figure 4. The curve that shows how the number of cases increases as the EID disaster progresses. ${ }^{52}$

3 Beyond time point of a crisis $\left(\mathrm{T}_{\mathrm{c}}\right)$, the medical facilities start to operate under a crisis mode.

4 In the early stage of an EID disaster, when the healthcare capacity is adequate, the DT models with high

5 sensitivity levels should be employed to identify patients with risk of infection progression for them to be

6 hospitalized and receive the best treatment ${ }^{9,43}$. In the later stages of an EID disaster, the available medical

7 resources may be exhausted due to a tremendous increase of patients. In this scenario, the DT models with a

8 lower sensitivity should be employed to recommend only those patients with a high risk of progressing to

9 severe infection or death for hospitalization and thereby avoid collapse of medical facilities. 


\section{Tables}

2 Table 1. Results of the first-stage feature selection by logistic regression

\begin{tabular}{|c|c|c|c|c|c|c|}
\hline Item & Variables ${ }^{*}$ & $\begin{array}{l}\text { All patients } \\
\mathrm{N}=83,227\end{array}$ & Estimate & Std. Error & $\mathrm{z}$ value & $\operatorname{Prob}(>|z|)$ \\
\hline 0 & (Intercept) & & -3.909283 & 0.037019 & -105.601 & $<0.000$ \\
\hline 1 & Age $($ mean \pm SD $)$ & $51.08 \pm 30.49$ & 0.031406 & 0.000562 & 55.882 & $<0.000$ \\
\hline 2 & Heart disease & $21,776(26.16 \%)$ & 0.448181 & 0.021741 & 20.615 & $<0.000$ \\
\hline 3 & Metastatic cancer & $4,264(5.12 \%)$ & 0.663727 & 0.037349 & 17.771 & $<0.000$ \\
\hline 4 & CVA & $16,935(20.35 \%)$ & 0.361054 & 0.022895 & 15.77 & $<0.000$ \\
\hline 5 & Renal disease & $6,617(7.95 \%)$ & 0.429659 & 0.029803 & 14.417 & $<0.000$ \\
\hline 6 & Neurological disease & $8,443(10.14 \%)$ & 0.36544 & 0.028077 & 13.016 & $<0.000$ \\
\hline 7 & Diabetes & $16,416(19.72 \%)$ & 0.280035 & 0.022435 & 12.482 & $<0.000$ \\
\hline 8 & Pulmonary disease & $32,646(39.23 \%)$ & -0.243137 & 0.021165 & -11.488 & $<0.000$ \\
\hline 9 & Gender (Male) & $49,739(59.76 \%)$ & 0.227208 & 0.020821 & 10.912 & $<0.000$ \\
\hline 10 & Hypertension & $30,663(36.84 \%)$ & -0.182299 & 0.02258 & -8.073 & $<0.000$ \\
\hline 11 & Tuberculosis & $3,083(3.7 \%)$ & 0.332174 & 0.042151 & 7.881 & $<0.000$ \\
\hline 12 & Cancer without metastasis & $4,113(4.94 \%)$ & 0.28759 & 0.038312 & 7.506 & $<0.000$ \\
\hline 13 & Leukemia and lymphoma & $922(1.11 \%)$ & 0.565562 & 0.082878 & 6.824 & $<0.000$ \\
\hline 14 & Pregnancy/ postpartum & $334(0.4 \%)$ & 1.006209 & 0.167368 & 6.012 & $<0.000$ \\
\hline 15 & PVD & $1,689(2.03 \%)$ & 0.253768 & 0.05406 & 4.694 & $<0.000$ \\
\hline 16 & AIDS & $63(0.08 \%)$ & 1.223306 & 0.306994 & 3.985 & $<0.000$ \\
\hline 17 & Severe liver disease & $289(0.35 \%)$ & 0.496771 & 0.142543 & 3.485 & 0.000 \\
\hline 18 & Autoimmune disease & $635(0.76 \%)$ & 0.262207 & 0.104528 & 2.508 & 0.012 \\
\hline 19 & Mental illness & $1,821(2.19 \%)$ & 0.100714 & 0.065314 & 1.542 & 0.123 \\
\hline 20 & Hypothyroidism & $558(0.67 \%)$ & 0.137819 & 0.100471 & 1.372 & 0.170 \\
\hline 21 & Hyperthyroidism & $542(0.65 \%)$ & 0.061082 & 0.112777 & 0.542 & 0.588 \\
\hline
\end{tabular}

3 *Abbreviations: CVA (cerebrovascular accident); PVD (peripheral vascular disease); AIDS (acquired immune

4 deficiency syndrome). 
1 Table 2. The F-1 scores delivered by the alternative prediction models with different feature sets

\begin{tabular}{|c|c|c|c|c|}
\hline Model & Feature set & $\begin{array}{c}\text { Sensitivity } 85 \% \\
\text { Mean( } 95 \% \text { confidence interval) }\end{array}$ & $\begin{array}{c}\text { Sensitivity } 90 \% \\
\text { Mean(95\% confidence interval) }\end{array}$ & $\begin{array}{c}\text { Sensitivity } 95 \% \\
\text { Mean(95\% confidence interval) }\end{array}$ \\
\hline \multirow{4}{*}{ DT } & $\begin{array}{l}6 \text { features } \\
\text { (by the proposed DT-based method) }\end{array}$ & $0.446(0.446-0.447)$ & $0.436(0.436-0.436)$ & $0.410(0.409-0.410)$ \\
\hline & $\begin{array}{l}6 \text { features } \\
\text { (by mRMRe) }\end{array}$ & $0.438(0.438-0.438)$ & $0.428(0.428-0.428)$ & $0.409(0.409-0.409)$ \\
\hline & \begin{tabular}{|l}
6 features \\
(by LASSO)
\end{tabular} & $0.437(0.437-0.437)$ & $0.428(0.427-0.428)$ & $0.400(0.400-0.400)$ \\
\hline & 18 features & $0.442(0.442-0.442)$ & $0.437(0.437-0.437)$ & $0.413(0.412-0.413)$ \\
\hline \multirow{4}{*}{ LR } & $\begin{array}{l}6 \text { features } \\
\text { (by the proposed DT-based method) }\end{array}$ & $0.440(0.440-0.441)$ & $0.430(0.430-0.430)$ & $0.399(0.399-0.400)$ \\
\hline & $\begin{array}{l}6 \text { features } \\
\text { (by mRMRe) }\end{array}$ & $0.435(0.435-0.435)$ & $0.423(0.423-0.424)$ & $0.398(0.398-0.399)$ \\
\hline & $\begin{array}{l}6 \text { features } \\
\text { (by LASSO) }\end{array}$ & $0.433(0.432-0.433)$ & $0.421(0.421-0.421)$ & $0.397(0.397-0.397)$ \\
\hline & 18 features & $0.446(0.446-0.446)$ & $0.434(0.434-0.434)$ & $0.406(0.406-0.406)$ \\
\hline \multirow{4}{*}{ DNN } & $\begin{array}{l}6 \text { features } \\
\text { (by the proposed DT-based method) }\end{array}$ & $0.447(0.447-0.447)$ & $0.437(0.437-0.437)$ & $0.409(0.408-0.409)$ \\
\hline & $\begin{array}{l}6 \text { features } \\
\text { (by mRMRe) }\end{array}$ & $0.438(0.438-0.438)$ & $0.431(0.430-0.431)$ & $0.410(0.410-0.410)$ \\
\hline & $\begin{array}{l}6 \text { features } \\
\text { (by LASSO) }\end{array}$ & $0.437(0.437-0.437)$ & $0.428(0.427-0.428)$ & $0.400(0.400-0.400)$ \\
\hline & 18 features & $0.452(0.451-0.452)$ & $0.444(0.443-0.444)$ & $0.422(0.421-0.422)$ \\
\hline
\end{tabular}

2 Note: Please refer to supplementary Table S5 for the definitions of the F1 score. 


\section{Footnotes}

2 Author Contributions: Conceived and designed this study: HYRC, CKH, SYC, FYS, HCH, CCK,

$3 \mathrm{CCF}$, and YJO. Analyzed the data: CKH, HYRC. Wrote this manuscript: HYRC, CKH, and SYC.

4 Revised this manuscript: FYS, CCF, JRG and YJO. All authors critically revised the manuscript and

5 agreed the final version.

6 Funding: We received no funding for this study

7 Competing interests: The authors have no competing interests as defined by Nature Research, or other

8 interests that might be perceived to influence the results and/or discussion reported in this paper.

9 Ethical approval: The study protocol was approved by the Research Ethics Committee of the National

10 Taiwan University Hospital (ID: 201603086RINB, April 14, 2016).

11 Transparency: On behalf of all authors, the lead author affirms that this manuscript is an honest, 12 accurate, and transparent account of the study being reported; that no important aspects of the study

13 have been omitted; and that any discrepancies from the study as planned (and, if relevant, registered)

14 have been explained.

15 Data sharing: All the de-identified data could be obtained from the National Health Insurance

16 Administration, Ministry of Health and Welfare in Taiwan, Republic of China (R. O. C.).

17 Acknowledgements: We deeply appreciate Mr. Chun-Hua Chang's efforts in the early stage of this

18 study, when he served in the Research Center for Applied Sciences, Academia Sinica, Taiwan. We also

19 deeply appreciate Dr. Ting-Chia Weng's efforts, when he served in the Department of Occupational

20 and Environmental Medicine, National Cheng Kung University Hospital, Taiwan. 


\section{References}

21 Lew, T. W. et al. Acute respiratory distress syndrome in critically ill patients with severe acute 3 respiratory syndrome. Jama 290, 374-380, doi:10.1001/jama.290.3.374 (2003).

42 Sullivan, S. J., Jacobson, R. M., Dowdle, W. R. \& Poland, G. A. 2009 H1N1 influenza. Mayo Clinic proceedings 85, 64-76, doi:10.4065/mcp.2009.0588 (2010).

63 Norris, S. L., Sawin, V. I., Ferri, M., Reques Sastre, L. \& Porgo, T. V. An evaluation of emergency guidelines issued by the World Health Organization in response to four infectious disease outbreaks. PloS one 13, e0198125, doi:10.1371/journal.pone.0198125 (2018).

Jiang, X. et al. Towards an artificial intelligence framework for data-driven prediction of coronavirus clinical severity. CMC-Computers, Materials \& Continua 63, 537-551 (2020).

Bavinger, J. C., Shantha, J. G. \& Yeh, S. Ebola, COVID-19, and emerging infectious disease: lessons learned and future preparedness. Current opinion in ophthalmology 31, 416-422, doi:10.1097/icu.0000000000000683 (2020).

Iacobucci, G. Covid-19: New UK variant may be linked to increased death rate, early data indicate. Bmj 372, n230, doi:10.1136/bmj.n230 (2021).

Gupta, M. et al. The need for COVID-19 research in low- and middle-income countries. Global health research and policy 5, 33, doi:10.1186/s41256-020-00159-y (2020).

Hollinghurst, J. et al. The impact of COVID-19 on adjusted mortality risk in care homes for older adults in Wales, UK: a retrospective population-based cohort study for mortality in 2016-2020. Age and ageing 50, 25-31, doi:10.1093/ageing/afaa207 (2021).

Butler, C. R., Wong, S. P. Y., Wightman, A. G. \& O'Hare, A. M. US Clinicians' Experiences and Perspectives on Resource Limitation and Patient Care During the COVID-19 Pandemic. JAMA Netw Open 3, e2027315, doi:10.1001/jamanetworkopen.2020.27315 (2020).

10 Novel, C. P. E. R. E. The epidemiological characteristics of an outbreak of 2019 novel coronavirus diseases (COVID-19) in China. Zhonghua liu xing bing xue za zhi= Zhonghua liuxingbingxue zazhi 41, 145 (2020).

Coccolini, F. et al. COVID-19 the showdown for mass casualty preparedness and management: the Cassandra Syndrome. World journal of emergency surgery : WJES 15, 26, doi:10.1186/s13017-020-00304-5 (2020).

12 Pourhomayoun, M. \& Shakibi, M. Predicting mortality risk in patients with COVID-19 using artificial intelligence to help medical decision-making. medRxiv (2020). 
113 Ho, T. S. et al. Comparing machine learning with case-control models to identify confirmed dengue cases. PLoS neglected tropical diseases 14, e0008843, doi:10.1371/journal.pntd.0008843 (2020).

414 Weng, S. F., Reps, J., Kai, J., Garibaldi, J. M. \& Qureshi, N. Can machine-learning improve cardiovascular risk prediction using routine clinical data? PloS one 12, e0174944 (2017).

615 James, G., Witten, D., Hastie, T. \& Tibshirani, R. An introduction to statistical learning. Vol. 112 (Springer, 2013).

Cortes, C. \& Vapnik, V. Support-vector networks. Machine learning 20, 273-297 (1995).

Cho, S., Hong, H. \& Ha, B.-C. A hybrid approach based on the combination of variable selection using decision trees and case-based reasoning using the Mahalanobis distance: For bankruptcy prediction. Expert Systems with Applications 37, 3482-3488 (2010).

18 Therneau, T. M., Atkinson, B. \& Ripley, M. B. The rpart package. (Oxford, UK, 2010).

19 Maimon, O. Z. \& Rokach, L. Data mining with decision trees: theory and applications. Vol. 81 (World scientific, 2014).

20 Sa-Ngamuang, C. et al. Accuracy of dengue clinical diagnosis with and without NS1 antigen rapid test: Comparison between human and Bayesian network model decision. PLoS neglected tropical diseases 12, e0006573 (2018).

21 Bai, J., Li, Y., Li, J., Jiang, Y. \& Xia, S. Rectified Decision Trees: Towards Interpretability, Compression and Empirical Soundness. arXiv preprint arXiv:1903.05965 (2019).

22 Siu, C. Automatic induction of neural network decision tree algorithms. in Intelligent ComputingProceedings of the Computing Conference. $697-704$ (Springer, 2019).

23 Marsden-Haug, N. et al. Code-based syndromic surveillance for influenzalike illness by International Classification of Diseases, Ninth Revision. Emerging infectious diseases 13, 207 (2007).

$24 \mathrm{Wu}, \mathrm{T}$. S. et al. Establishing a nationwide emergency department-based syndromic surveillance system for better public health responses in Taiwan. BMC Public Health 8, 18, doi:10.1186/14712458-8-18 (2008).

25 Wu, T. S. Establishing Emergency Department-Based Infectious Disease Syndromic Surveillance System in Taiwan-Aberration Detection Methods, Epidemiological Characteristics, System Evaluation and Recommendations Master Degree Thesis thesis, National Taiwan University (2006). 
126 Weng, T. C. et al. National retrospective cohort study to identify age-specific fatality risks of comorbidities among hospitalised patients with influenza-like illness in Taiwan. BMJ open 9, e025276, doi:10.1136/bmjopen-2018-025276 (2019).

Charlson, M., Szatrowski, T. P., Peterson, J. \& Gold, J. Validation of a combined comorbidity index. Journal of clinical epidemiology 47, 1245-1251 (1994).

28 Quan, H. et al. Coding algorithms for defining comorbidities in ICD-9-CM and ICD-10 administrative data. Med Care 43, 1130-1139, doi:10.1097/01.mlr.0000182534.19832.83 (2005).

29 Deyo, R. A., Cherkin, D. C. \& Ciol, M. A. Adapting a clinical comorbidity index for use with ICD-9-CM administrative databases. Journal of clinical epidemiology 45, 613-619 (1992).

30 Elixhauser, A., Steiner, C., Harris, D. R. \& Coffey, R. M. Comorbidity measures for use with administrative data. Medical care, 8-27 (1998).

31 Muthukrishnan, R. \& Rohini, R. LASSO: A feature selection technique in predictive modeling for machine learning. in 2016 IEEE international conference on advances in computer applications (ICACA). 18-20 (IEEE, 2016).

32 Zhao, Z., Anand, R. \& Wang, M. Maximum relevance and minimum redundancy feature selection methods for a marketing machine learning platform. in 2019 IEEE International Conference on Data Science and Advanced Analytics (DSAA). 442-452 (IEEE, 2019).

33 De Jay, N. et al. mRMRe: an R package for parallelized mRMR ensemble feature selection. Bioinformatics 29, 2365-2368 (2013).

34 Agresti, A. An introduction to categorical data analysis. (John Wiley \& Sons, 2018).

35 Fushiki, T. Estimation of prediction error by using K-fold cross-validation. Statistics and Computing 21, 137-146 (2011).

36 Desai, V. S., Crook, J. N. \& Overstreet Jr, G. A. A comparison of neural networks and linear scoring models in the credit union environment. European journal of operational research 95, 2437 (1996).

37 Dreiseitl, S. \& Ohno-Machado, L. Logistic regression and artificial neural network classification models: a methodology review. Journal of biomedical informatics 35, 352-359 (2002).

8 Tharwat, A. Classification assessment methods. Applied Computing and Informatics (2020).

39 Munsch, N. et al. Diagnostic Accuracy of Web-Based COVID-19 Symptom Checkers: Comparison Study. Journal of medical Internet research 22, e21299, doi:10.2196/21299 (2020). 
140 Kotsiantis, S. B., Zaharakis, I. \& Pintelas, P. Supervised machine learning: A review of classification techniques. Emerging artificial intelligence applications in computer engineering 160, 3-24 (2007).

Premkumar, M. et al. A/H1N1/09 Influenza is Associated With High Mortality in Liver Cirrhosis. Journal of clinical and experimental hepatology 9, 162-170, doi:10.1016/j.jceh.2018.04.006 (2019).

2 Chowell, G., Ayala, A., Berisha, V., Viboud, C. \& Schumacher, M. Risk factors for mortality among 2009 A/H1N1 influenza hospitalizations in Maricopa County, Arizona, April 2009 to March 2010. Computational and mathematical methods in medicine 2012, 914196, doi:10.1155/2012/914196 (2012).

43 Laventhal, N. et al. The Ethics of Creating a Resource Allocation Strategy During the COVID-19 Pandemic. Pediatrics 146, doi:10.1542/peds.2020-1243 (2020).

44 White, D. B., Katz, M. H., Luce, J. M. \& Lo, B. Who should receive life support during a public health emergency? Using ethical principles to improve allocation decisions. Annals of internal medicine 150, 132-138, doi:10.7326/0003-4819-150-2-200901200-00011 (2009).

45 Hussain, A., Mahawar, K., Xia, Z., Yang, W. \& El-Hasani, S. Obesity and mortality of COVID19. Meta-analysis. Obesity research \& clinical practice 14, 295-300, doi:10.1016/j.orcp.2020.07.002 (2020).

46 Yang, L. et al. Obesity and influenza associated mortality: evidence from an elderly cohort in Hong Kong. Preventive medicine 56, 118-123 (2013).

47 Sanchez-Ramirez, D. C. \& Mackey, D. Underlying respiratory diseases, specifically COPD, and smoking are associated with severe COVID-19 outcomes: A systematic review and meta-analysis. Respiratory medicine 171, 106096, doi:10.1016/j.rmed.2020.106096 (2020).

48 Team, C. C.-R. et al. Geographic differences in COVID-19 cases, deaths, and incidence-United States, February 12-April 7, 2020. Morbidity and Mortality Weekly Report 69, 465-471 (2020).

49 Shadmi, E. et al. Health equity and COVID-19: global perspectives. International journal for equity in health 19, 104, doi:10.1186/s12939-020-01218-z (2020).

50 Arabi, Y. M., Fowler, R. \& Hayden, F. G. Critical care management of adults with communityacquired severe respiratory viral infection. Intensive care medicine 46, 315-328, doi:10.1007/s00134-020-05943-5 (2020).

1 Uyeki, T. M. Influenza. Annals of internal medicine 167, Itc33-itc48, doi:10.7326/aitc201709050 (2017). 
152 Casani, J. A. P. et al. Surge capacity. Disaster Medicine, 193 (2006). 


\section{Supplementary Files}

This is a list of supplementary files associated with this preprint. Click to download.

- SubmissionILIMLSupplementaryMaterial20210809Final.pdf 